\title{
Iron-Based Superconductors as Odd-Parity Superconductors
}

\author{
Jiangping $\mathrm{Hu}^{1,2}$ \\ ${ }^{1}$ Beijing National Laboratory for Condensed Matter Physics, Institute of Physics, Chinese Academy of Sciences, \\ Beijing 100080, China \\ ${ }^{2}$ Department of Physics, Purdue University, West Lafayette, Indiana 47907, USA
}

(Received 28 February 2013; revised manuscript received 26 March 2013; published 16 July 2013)

\begin{abstract}
Parity is a fundamental quantum number used to classify a state of matter. Materials rarely possess ground states with odd parity. We show that the superconducting state in iron-based superconductors is classified as an odd-parity $s$-wave spin-singlet pairing state in a single trilayer FeAs/Se, the building block of the materials. In a low-energy effective model constructed on the Fe square bipartite lattice, the superconducting order parameter in this state is a combination of an $s$-wave normal pairing between two sublattices and an $s$-wave $\eta$ pairing within the sublattices. The state has a fingerprint with a real-space sign inversion between the top and bottom As/Se layers. The results suggest that iron-based superconductors are a new quantum state of matter, and the measurement of the odd parity can help to establish high-temperature superconducting mechanisms.
\end{abstract}

DOI: 10.1103/PhysRevX.3.031004

\section{INTRODUCTION}

Symmetry plays the central role in the search for beauty in physics. It controls the structure of matter and allows us to simplify a complicated problem. The gauge principle is a fundamental principle in physics. Models formulated in different gauge settings are equivalent. Symmetry and gauge principles together provide the foundations of modern physics that allow us to solve complicated problems.

The recently discovered iron-based high-temperature superconductors (high $T_{c}$ ) [1-3] are layered materials with complicated electronic structures. Their complexity causes a major difficulty in understanding pairing symmetry, which arguably is the most important property and clue to determine the pairing mechanism $[4,5]$.

In a strongly correlated electron system, major physics is determined locally in real space. Important properties, such as pairing symmetry in a superconducting state, are expected to be robust against small variations of Fermi surfaces in reciprocal space. Although the superconducting mechanism related to high-temperature superconductors (high $T_{c}$ ) has not yet been determined, the robust $d$-wave pairing symmetry in cuprates [6] can be understood under this principle.

Does this principle still hold for iron-based superconductors? Namely, do all iron-based superconductors possess one universal pairing state? Unlike cuprates, the answer to this question is highly controversial because different theoretical approaches have provided different answers and no universal state has been identified [5]. Nevertheless, as local electronic structures in all families

Published by the American Physical Society under the terms of the Creative Commons Attribution 3.0 License. Further distribution of this work must maintain attribution to the author(s) and the published article's title, journal citation, and DOI.
Subject Areas: Condensed Matter Physics, Superconductivity

of iron-based superconductors are almost identical and phase diagrams are smooth against doping [4,5], it is hard to argue that the materials can approach many different superconducting ground states.

According to conventional wisdom, there are several obvious requirements regarding electron pairing in superconducting states. First, pairing symmetry is known to be classified according to lattice symmetry. Second, in a uniform superconducting state, the total momentum for the Cooper pairs (modulo a reciprocal lattice vector) must vanish. Finally, for a central symmetric lattice with a space-inversion center, the parity of superconducting order parameters is normally even for a spin-singlet pairing and odd for a spin-triplet pairing [7]. These requirements are easily fulfilled in a system with a simple electronic structure, such as cuprates. However, for iron-based superconductors, they are highly nontrivial.

The unit cell in iron-based superconductors is intrinsically a 2-Fe unit cell, while, for simplicity, most theoretical models are effectively constructed based on a 1-Fe unit cell [8-13]. Obviously, these effective models have different lattice symmetry than the models defined on the original lattice. The difference may cause serious problems. For example, in the effective models, the pairings have been limited to two electrons with opposite momentum $(\vec{k},-\vec{k})$, which we call normal pairing in this paper, where $\vec{k}$ is the momentum defined with respect to the 1-Fe unit cell in an iron-square lattice. The momentum vector $Q=(\pi, \pi)$ is a reciprocal lattice vector in the original lattice with a $2-\mathrm{Fe}$ unit cell. Thus, the pairings $(\vec{k},-\vec{k}+Q)$, in principle, are also allowed according to the second requirement above. We will refer to this pairing channel as an extended $\eta$ pairing $[14,15]$ and simply call it $\eta$ pairing in this paper. The possible existence of $\eta$ pairing was discussed in simplified models [16-18]. As order parameters are classified differently under different symmetry groups, we 
need to understand these orders under the original lattice symmetry. Otherwise, conservation laws could be violated.

In this paper, we show that the superconducting state in iron-based superconductors is a new state of matter that is classified as an odd-parity $s$-wave spin-singlet pairing state in a single trilayer FeAs/Se, which is the building block of the materials. Provided that essential physics stems from $d-p$ hybridization, the superconducting states that were proposed in the past based on the effective $d$-orbital models of an iron-square lattice are not parity eigenstates. Namely, parity conservation is violated. We show that the superconducting state that conserves parity includes both normal pairing between two sublattices of the iron-square lattice and $\eta$ pairing within each sublattice. The states have a fingerprint with a real-space sign inversion between the top and bottom As/Se layers. Our derivation is based on general symmetry and gauge requirements on the effective models for iron-based superconductors.

In the following, we first provide a complete symmetry analysis for pairing symmetries in iron-based superconductors. While the pairing symmetries can be classified according to $D_{2 d}$-point groups at iron sites or $C_{4 v}$-point groups at the center of an iron square, there are two types of pairing symmetries for a spin-singlet pairing state because of the intrinsic 2-Fe unit cell. They are distinguished from each other by opposite parity numbers. Second, we discuss the hidden symmetry properties of the effective models under the original lattice symmetry. We show that the effective hopping terms between two sublattices and within each sublattice have different symmetry characters. Third, we discuss a general gauge principle related to the definition of pairing symmetries and conclude that parity conservation was violated in the past. We provide the mean-field Hamiltonian in the new superconducting state and show that it can provide a unified description of all families of iron-based superconductors, including both iron-pnictides [4] and iron-chalcognides [19-22]. Fourth, we discuss the smoking-gun experiments that can reveal the parity of the superconducting state. Finally, we discuss the fundamental impact on the high- $T_{c}$ superconducting mechanism, if it is confirmed.

Before we start the main part of this paper, we first clarify the gauge setting for the effective models that were constructed based on all five iron $d$ orbitals [8-10]. In those effective models with a 1-Fe unit cell, a new gauge setting is taken [8-10], which effectively changes the momentum $\vec{k}$ to $\vec{k}+Q$ for $d_{x z}$ and $d_{y z}$ orbitals. In the following, without further clarification, the momentum $\vec{k}$ used in the definition of our normal pairing $(\vec{k},-\vec{k})$ and $\eta$ pairing $(\vec{k},-\vec{k}+Q)$ is the same momentum used in those papers, rather than the momentum in a natural gauge setting in which Wannier functions of each orbital at $\mathrm{Fe}$ sites are translationally invariant with respect to $1-\mathrm{Fe}$ unit cells and have the same symmetry classification as atomic $d$ orbitals.

\section{SYMMETRY OF A SINGLE FE-AS(SE) TRILAYER}

Iron-based superconductors are layered materials. The essential electronic physics is controlled by a single trilayer $\mathrm{Fe}-\mathrm{As}(\mathrm{Se})$ structure, which is the building block of the superconductors. Although the coupling along the $c$ axis between the building blocks has many interesting effects, the superconducting mechanism and the fundamental properties of the superconducting states, such as pairing symmetries, are expected to be solely determined within the single building block. The observation of superconductivity in a single FeSe layer grown by molecular beam epitaxy has further justified this two-dimensional nature [20-22]. Therefore, we first focus on the study of a single $\mathrm{Fe}-\mathrm{As}(\mathrm{Se})$ trilayer structure.

We start our analysis by understanding the lattice symmetry. As shown in Fig. 1, the structure has an inversion center, the origin, located in the middle of each Fe-Fe link. The unit cell with its origin at the center is marked by the shadowed area, which includes two iron and two As/Se atoms. We denote $T$ as the translation group with respect to the unit cell. The symmetry group is thus described by a nonsymmorphic space group $G=P 4 / \mathrm{nmm}$ [23]. The quotient group $G / T$ is specified by 16 symmetry operations that include a space inversion $\hat{I}$. It is easy to check that these operations can be specified equivalently as $C_{4 v} \oplus$ $\hat{I} C_{4 v}$ or $D_{2 d} \oplus \hat{I} D_{2 d}$, where $C_{4 v}$ is the point group with respect to the point in the middle of an iron square and $D_{2 d}$ is the point group defined at an iron site. It is important to note that neither $C_{4 v}$ nor $D_{2 d}$ is defined with respect to the inversion center. Therefore, some symmetry operations in $C_{4 v}$ or $D_{2 d}$ are nonsymmorphic. For example, the $\hat{C}_{4}$ rotation operation in $C_{4 v}$ is equivalent to $\left(\hat{C}_{4}^{\prime}, \hat{t}_{2}\right)$, which represents $\hat{C}_{4}^{\prime}$, a rotation $\frac{\pi}{2}$ along the $z$ axis at the inversion center, followed by a translation operation $\hat{t}_{2}$ that translates

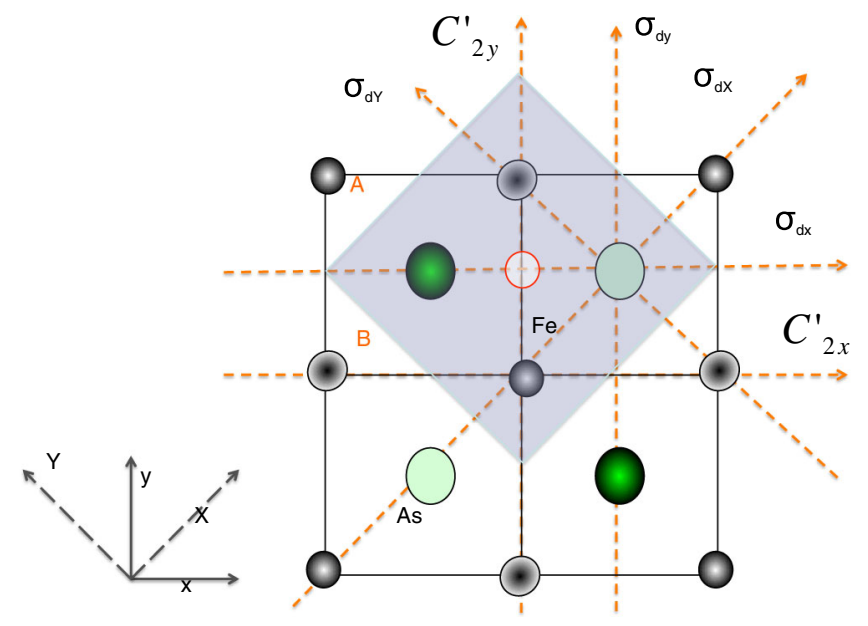

FIG. 1. Sketch of the lattice structure of a trilayer Fe-As(Se) unit. Notations used in this paper for axis directions, reflection symmetries, and two sublattices are noted. 
$\left(\frac{1}{2}, \frac{1}{2}, 0\right)$ in the coordinate of an iron-square lattice, which is half of a unit lattice cell along the $X$ direction in Fig. 1 .

In summary, the full symmetry group can be written as

$$
G / T=Z_{2} \otimes D_{2 d}=Z_{2} \otimes C_{4 v},
$$

where $Z_{2}=(\hat{E}, \hat{I})$. The group is a direct product of two subgroups that are defined with respect to different operation centers. $\hat{I}$ commutates with all symmetry operations in $D_{2 d}$ or $C_{4 v}$, in the sense that operations are considered to be identical if they only differ by a lattice transition with respect to the 2-Fe unit cell.

\section{PARITY AND PAIRING SYMMETRY CLASSIFICATION}

The pairing symmetry of a translation-invariant superconducting state is classified by the irreducible representations (IRs) of $G / T$. If we ignore spin-orbital coupling, the ground state is expected to be a parity eigenstate. Since spin-singlet pairing is overwhelmingly supported experimentally in iron-based superconductors [4,5], we focus on spin-singlet pairing states.

Conventionally, for a spin-singlet pairing state, the parity is even. However, because of the unit cell doubling, the parity operation here essentially takes mapping between two sublattices, $A$ and $B$, in the iron-square lattice, as shown in Fig. 1. Therefore, there is no parity constraint for the pairing within each sublattice governed by $D_{2 d}$. Thus, for each IR of $D_{2 d}$, there are two different pairing states with opposite parities. The IRs of $G / T$ are direct products of the IRs of two subgroups.

The character table of the $D_{2 d}$ group is shown in Table. I. There are five different IRs, with four being one dimensional $(A$ and $B$ ) and one being two dimensional $(E)$. Let us consider the symmetry operation $\hat{S}_{4}^{2}=\hat{S}_{4} \times \hat{S}_{4}$ in $D_{2 d}$. It is easy to show that

$$
\hat{I} \hat{S}_{4}^{2}=\left(\hat{\sigma}_{h}, \hat{t}_{2}^{\prime}\right),
$$

where $\hat{\sigma}_{h}$ is the reflection along the $z$ axis and $\hat{t}_{2}^{\prime}$ is an inplane translation by $(1,0,0)$, namely, one iron-iron lattice distance. Equation (2) leads to an extremely important conclusion: The parity is determined by the eigenvalues of the operation $\left(\hat{\sigma}_{h}, \hat{t}_{2}^{\prime}\right)$. It is equal or opposite to the eigenvalues for one-dimensional $(A$ and $B)$ or twodimensional $(E)$ IRs, respectively, because $\hat{S}_{4}^{2}=1$ for one-dimensional IRs and $\hat{S}_{4}^{2}=-1$ for two-dimensional

TABLE I. Character table for $D_{2 d}$ point group.

\begin{tabular}{rrrrrrcc}
\hline \hline & $E$ & $2 S_{4}$ & $C_{2}(z)$ & $2 C_{2}^{\prime}$ & $2 \sigma_{d}$ & Linear, rotations & Quadratic \\
\hline$A_{1}$ & 1 & 1 & 1 & 1 & 1 & & $x^{2}+y^{2}, z^{2}$ \\
$A_{2}$ & 1 & 1 & 1 & -1 & -1 & $R_{z}$ & \\
$B_{1}$ & 1 & -1 & 1 & 1 & -1 & & $x^{2}-y^{2}$ \\
$B_{2}$ & 1 & -1 & 1 & -1 & 1 & $z$ & $x y$ \\
$E$ & 2 & 0 & -2 & 0 & 0 & $(x, y)\left(R_{x}, R_{y}\right)$ & $(x z, y z)$ \\
\hline \hline
\end{tabular}

IRs. More specifically, this result leads to the conclusion that the pairing is translationally invariant with respect to a 1-Fe unit cell in the $A$ or $B$ state when the parity is even and in the $E$ state when the parity is odd. The $\eta$ pairing takes place in the $A$ or $B$ state with odd parity and in the $E$ state with even parity.

The above classification is independent of the number of orbitals and orbital characters as long as we have the gauge setting specified for $d_{x z}$ and $d_{y z}$ as mentioned earlier.

It is also important to note that the classifications with respect to $D_{2 d}$ at iron sites or $C_{4 v}$ at the center of iron squares are equivalent, in the sense that they can be mapped to each other. $C_{4 v}$ has the same number and type of IRs as $D_{2 d}$. We notice the following important relation,

$$
\hat{S}_{4}^{3}=\hat{I} \hat{C}_{4},
$$

where $\hat{C}_{4}$ is the $\pi / 2$ rotation operation in $C_{4 v}$. For onedimensional IRs in $D_{2 d}$, the above equation reduces to $\hat{S}_{4}=\hat{I} \hat{C}_{4}$. Therefore, for parity-even pairing, namely, normal pairing, there is no difference whether states are classified according to $D_{2 d}$ or $C_{4 v}$ since $\hat{S}_{4}=\hat{C}_{4}$. Namely, a normal pairing state has the same IRs with respect to both $C_{4 v}$ and $D_{2 d}$. For parity-odd $\eta$ pairing, $\hat{S}_{4}=-\hat{C}_{4}$, which implies that an $s$-wave state classified by $A$ IRs in $D_{2 d}$ must become a $d$-wave state classified by $B$ IRs in $C_{4 v}$. For example, an $\eta$-pairing $A_{1} s$-wave state classified in $D_{2 d}$ belongs to the $B_{2} d$ wave in $C_{4 v}$. Therefore, for an $\eta$-pairing parity-odd state, the name of the state depends on how it is classified. For the above example, one can either name the $\eta$-pairing state as a $B_{2 u} d$ wave or an $A_{1 u} s$ wave, depending on the classification point groups $C_{4 v}$ or $D_{2 d}$, respectively.

An odd-parity superconducting state must have a sign change between the top and bottom As/Se layers. However, this information is hidden in an effective model with only $d$ orbitals constructed on an iron-square lattice. From the above symmetry analysis, we can track the parity information simply by using $\hat{\sigma}_{h}$. Although $\hat{\sigma}_{h}$ is not a symmetry operation for a single $\mathrm{Fe}-\mathrm{As}(\mathrm{Se})$ trilayer, the $\eta$-pairing state can be viewed as a state with an internal negative isospin defined by $\hat{\sigma}_{h}$. For any normal pairing, $\hat{\Delta}^{n}$ and $\eta$-pairing $\hat{\Delta}^{\eta}$ order parameters that belong to onedimensional IRs of $D_{2 d}$, we have

$$
\begin{gathered}
\hat{\sigma}_{h} \hat{\Delta}^{n} \hat{\sigma}_{h}=\hat{\Delta}^{n}, \\
\hat{\sigma}_{h} \hat{\Delta}^{\eta} \hat{\sigma}_{h}=-\hat{\Delta}^{\eta} .
\end{gathered}
$$

We can extend the above discussion for order parameters in a bulk material. There are two different lattice structures along the $c$ axis in iron-based superconductors, the 11 type [which includes 111(NaFeAs) and 1111(LaOFeAs) structures] and the 122 type, where the 11 type is translationally invariant along the $c$ axis while the 122 type is not. 
For the 11 type, we can simply extend the above analysis to the nearest-neighbor (NN) interlayer pairing. For evenparity order parameters, we have two possible terms: $(\vec{k},-\vec{k})$ pairing, which is proportional to $\cos \left(k_{z}\right)$, and $\eta$ pairing $(\vec{k},-\vec{k}+Q)$, which is proportional to $\operatorname{isin}\left(k_{z}\right)$. For odd-parity order parameters, the two possible terms become $(\vec{k},-\vec{k})$ pairing, which is proportional to $\operatorname{isin}\left(k_{z}\right)$, and $\eta$,pairing $(\vec{k},-\vec{k}+Q)$, which is proportional to $\cos \left(k_{z}\right)$. Both terms can be in the same irreducible representations of $D_{2 d}$.

For the 122 type, the situation is rather different because the 122 structure has a symmorphic space group $I 4 / \mathrm{mmm}$ with a point group $D_{4 h}$ centered in the middle of two NN $\mathrm{Fe}-\mathrm{As}(\mathrm{Se})$ layers. The translation symmetry is specified by $(1,1,0),(0,1,1)$, and $(0,1,1)$. The space inversion and $\hat{\sigma}_{h}$ have identical characters in any one-dimensional IRs. A state with odd parity that belongs to one-dimensional IRs must have node lines on Fermi surfaces when $k_{z}=0$. Therefore, we are only allowed to construct even-parity states. For the intralayer pairing, there are two different even-parity order parameters: One is constructed by $(\vec{k},-\vec{k})$ pairing, and the other is constructed by $\left(\vec{k},-\vec{k}+Q_{3}\right)$ pairing, where $Q_{3}=(\pi, \pi, \pi)$, namely, $\eta$ pairing. The difference between these two order parameters is that the latter breaks $(1,0,1)$ and $(0,1,1)$ translation symmetry. Now, if we consider the NN interlayer pairing, we can have two terms that are parity even and keep the translation symmetry: the normal pairing $(\vec{k},-\vec{k})$, which is proportional to $\cos \left(k_{z}\right)$, and $(\vec{k},-\vec{k}+Q)$ pairing, which is proportional to $\operatorname{isin}\left(k_{z}\right)$. Here, we assume $Q=(\pi, \pi, 0)$. For the $\left(\vec{k},-\vec{k}+Q_{3}\right) \eta$ pairing, there are also two terms in the NN interlayer pairing: $\eta$ pairing $\left(\vec{k},-\vec{k}+Q_{3}\right)$ proportional to $\cos \left(k_{z}\right)$ and $(\vec{k},-\vec{k}+Q)$ pairing proportional to $\cos \left(k_{z}\right)$. Therefore, if the interlayer pairing is included, the superconducting state generally breaks the translation symmetry of the iron-square lattice.

\section{EFFECTIVE HAMILTONIAN AND HIDDEN SYMMETRY}

The above symmetry analysis is based on the original lattice symmetry. As we mentioned above, an effective model based on $d$ orbitals appears to have a different symmetry. In the past studies, we treated the model in a 1-Fe unit cell with a $D_{4 h}$ point group at iron sites. The treatment, in general, violated the basic spirit of the symmetry principle and might have resulted in fundamental errors. To fully respect the symmetry principle, we must understand the symmetry properties of the effective model under the original lattice symmetry.

We consider a general Hamiltonian in a single trilayer $\mathrm{Fe}-\mathrm{As}(\mathrm{Se})$ structure coordinated by $\mathrm{Fe}$ and $\mathrm{As}(\mathrm{Se})$ atoms,

$$
\hat{H}=\hat{H}_{d d}+\hat{H}_{d p}+\hat{H}_{p p}+\hat{H}_{I}
$$

where $\hat{H}_{d d}, H_{d p}$, and $\hat{H}_{p p}$ describe the direct hopping between two $d$ orbitals- the $d-p$ hybridization between $\mathrm{Fe}$ and $\mathrm{As}(\mathrm{Se})$ and the direct hopping between two $p$ orbitals, respectively. $\hat{H}_{I}$ describes any standard interactions. Here, we do not need to specify the parameter details. This Hamiltonian has a full symmetry defined by the nonsymmorphic space group.

An effective Hamiltonian is obtained by integrating out $p$ orbitals, which can be written as

$$
\hat{H}_{\mathrm{eff}}=\hat{H}_{d d, \text { eff }}+\hat{H}_{I, \mathrm{eff}} .
$$

The effective band structure can be written as $\hat{H}_{d d \text {,eff }}=$ $\hat{H}_{d d}+\hat{H}_{d p d}$, where $\hat{H}_{d p d}$ is the effective hopping induced through $d$ - $p$ hybridization. $\hat{H}_{d d \text {,eff }}$ has been obtained by many groups [8-10,24,25]. The major effective hopping terms in $\hat{H}_{d p d}$ can be divided into two parts: $\hat{H}_{d p d, \mathrm{NN}}$, which describes NN hopping, and $\hat{H}_{d p d, \mathrm{NNN}}$, which describes NNN hopping in the iron-square lattice. If one carefully checks the effective hopping parameters for $t_{2 g}$ orbitals in $\hat{H}_{d p d, \mathrm{NN}}$, one finds that they have the opposite sign from what we normally expect in a natural gauge setting, as shown in Figs. 2(a) and 2(b) where the $d_{x y}$ orbital is illustrated as an example. We see that the hopping parameter $t_{d d}$ must be negative. However, the effective hopping parameter $t_{d p d}$ is positive and even larger than

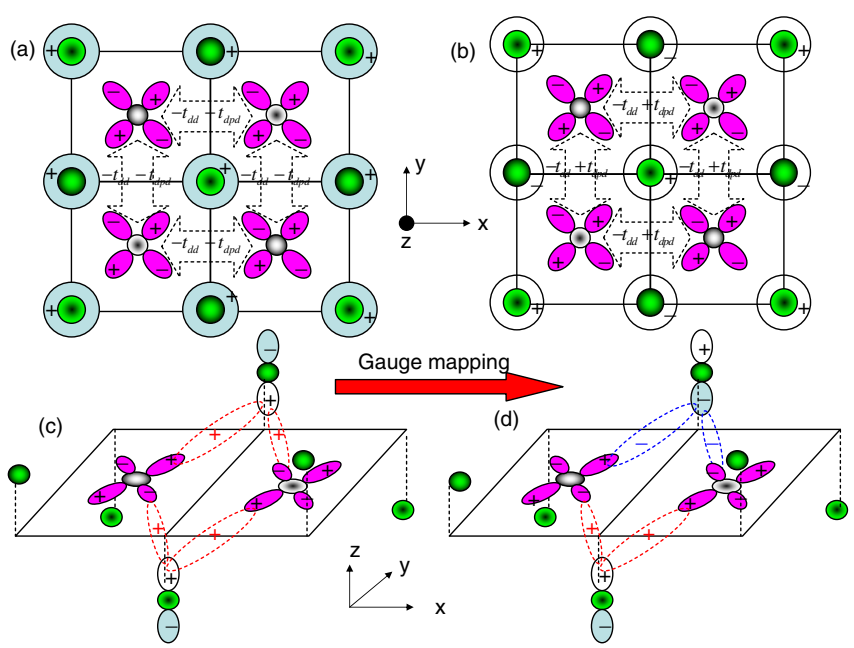

FIG. 2. The nearest-neighbor hopping parameters for intra- $d_{x y}$ orbitals are shown in (a) and (b). $t_{d d}$ is the amplitude of the direct hopping of $d_{x y}$ orbitals, while $t_{d p d}$ is the amplitude of the indirect hopping through $p_{z}$ orbitals of As/Se atoms. The reason for the sign change in $t_{d p d}$ between (a) and (b) is that the $p_{z}$ orbitals in the top layer and the bottom layer form occupied bonding states in (a) and empty antibonding states in (b). The difference is illustrated by the filled and empty $p_{z}$ orbitals in (a) and (b). (c) and (d) show the local $p-d s$-wave pairing pattern and a gauge transformation between them, which is defined by adding a minus sign to all $p_{z}$ orbitals on the top $\mathrm{As}(\mathrm{Se})$ layer but not to those on the bottom layer. 
$\left|t_{d d}\right|$ in $[8-10,24,25]$. In a tetragonal lattice, $t_{d p d}$ can only be generated through $d_{x y}-p_{z}$ hybridization. A positive value of $t_{d p d}$ suggests that virtual hopping that generates $t_{d p d}$ must go through an unoccupied $p_{z}$ state. As shown in Figs. 2(a) and 2(b), a $d_{x y}$ equally couples to $p_{z}$ orbitals of top and bottom As atoms. A high-energy $p_{z}$ state must be an antibonding $p_{z}$ state between NN As atoms. This analysis is held for all $t_{2 g}$ orbitals that play the dominant role in low-energy physics. It is also easy to check that the effective NNN hoppings between $t_{2 g}$ orbitals are dominated by occupied $p$ states, which are primarily bonding states of $p$

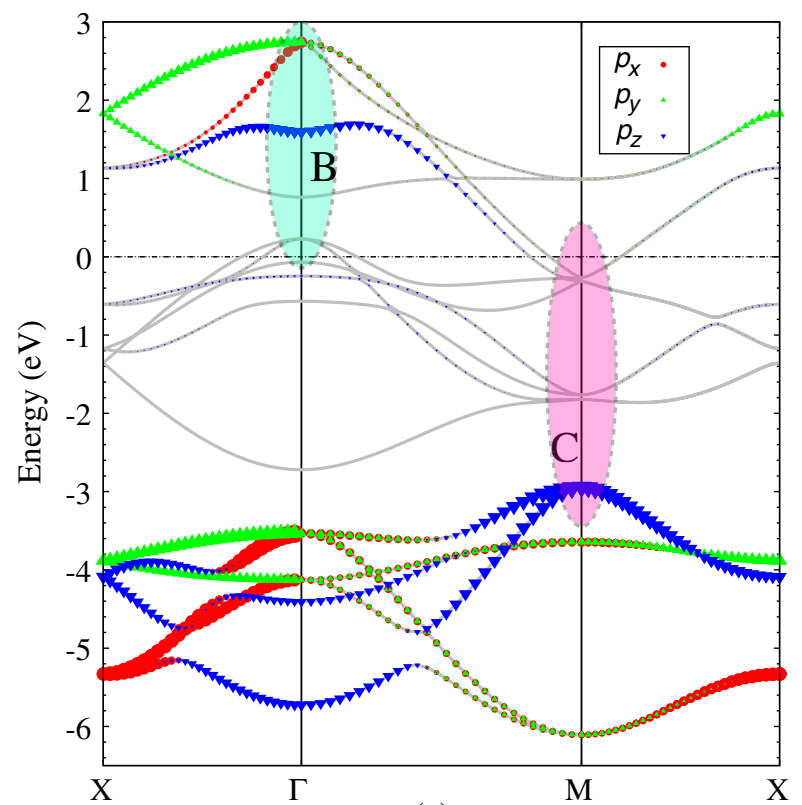

(a)

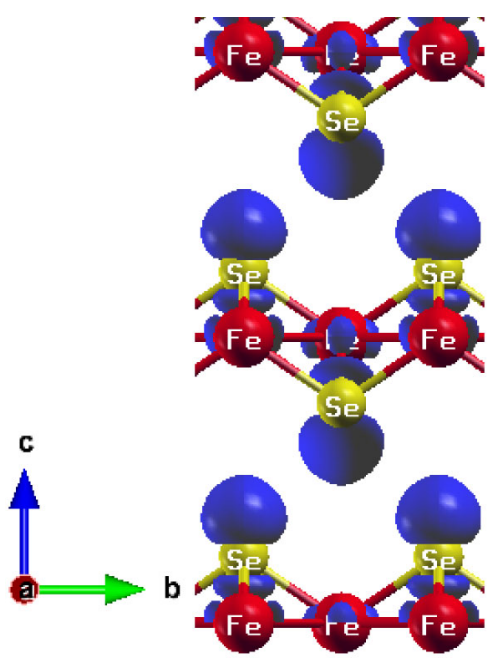

(b)

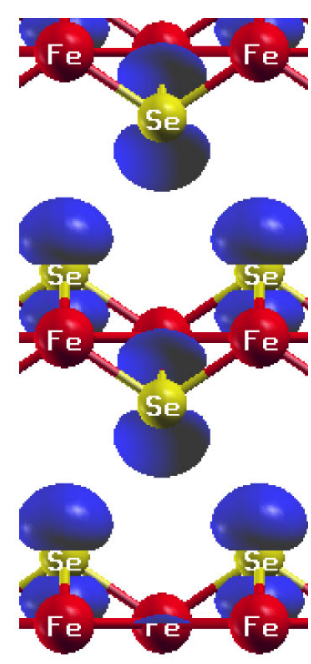

(c)
FIG. 3. (a) The calculated band structure of FeSe with the weight of $p$ orbitals of Se. (b) The decomposed charge density of the band at $\Gamma$ marked by the letter B (antibonding states). (c) The decomposed charge density of the band at $M$ marked by the letter $\mathrm{C}$ (bonding states). orbitals. Therefore, the NN effective hoppings are generated through $d-p_{a}$ hybridization, where $p_{a}$ represents an antibonding $p$-orbital state and the NNN effective hoppings are generated through $d-p_{b}$ hybridization, where $p_{b}$ is the bonding $p$ state.

The above microscopic understanding is not surprising. In fact, it is known in LDA calculations $[24,26,27]$ that $p$ orbitals in As/Se are not fully occupied, and there is significant overlap between $p$ orbitals on the bottom and top As/Se layers. Moreover, since $\hat{H}_{d p d, \mathrm{NN}}$ and $\hat{H}_{d p d, \mathrm{NNN}}$ primarily affect hole pockets around $\Gamma$ and electron pockets at $M$, respectively, we can check the distribution of antibonding $p$ states and bonding $p$ states in the band structure to further confirm the analysis. In Fig. 3(a), we plot the band structure of FeSe and the distribution of $p$ orbitals. The $p_{z}$ orbitals of Se are mainly at $+1.5 \mathrm{eV}$ at $\Gamma$ and $-3 \mathrm{eV}$ at $M$. By analyzing the bands at $\Gamma$ and $M$, as shown in Figs. 3(b) and 3(c), we confirm that the $p_{z}$ orbitals of Se at $\Gamma$ and $M$ belong to antibonding and bonding states, respectively.

Knowing the above hidden microscopic origins in the derivation of an effective Hamiltonian allows us to understand the characters of the effective Hamiltonian in the original lattice symmetry.

The $d-p_{a}$ hybridization is odd under $\hat{\sigma}_{h}$, while the $d-p_{b}$ hybridization is even under $\hat{\sigma}_{h}$. Thus, the NN hopping $\hat{H}_{d p d, \mathrm{NN}}$ and NNN hopping $\hat{H}_{d p d, \mathrm{NNN}}$ should be classified as odd and even under $\hat{\sigma}_{h}$, respectively. Namely,

$$
\begin{gathered}
\hat{\sigma}_{h} \hat{H}_{d p d, \mathrm{NN}} \hat{\sigma}_{h}=-\hat{H}_{d p d, \mathrm{NN}}, \\
\hat{\sigma}_{h} \hat{H}_{d p d, \mathrm{NNN}} \hat{\sigma}_{h}=\hat{H}_{d p d, \mathrm{NNN}} .
\end{gathered}
$$

The above hidden symmetry property is different than the main assumption taken in many weak-coupling approaches, which assume that the essential physics is driven by the interplay between hole pockets at $\Gamma$ and electron pockets at $M$ [5]. As indicated in Fig. 3(a), the interplay between the hole and electron pockets must be minimal because of their distinct microscopic origins.

\section{GAUGE PRINCIPLE AND PARITY CONSERVATION}

The symmetry difference in Eq. (8) has a fundamental impact on how we consider the parity of a superconducting state if superconducting pairing is driven by local $d$-p hybridization.

It has been shown that in a system where short-range pairings in real space dominate, superconducting order parameters are momentum dependent and a gauge principle must be satisfied because the phases of superconducting order parameters can be exchanged with those of the local hopping parameters [16,28] by gauge transformations. As an example, a $d$-wave superconducting state in cuprates can be mapped to an $s$-wave superconducting state by a gauge mapping that changes the hopping terms 
from $s$-type symmetry to $d$-type symmetry [29]. Therefore, only the combined symmetry of hopping terms and their associated pairing orders is gauge independent and can be used to classify superconducting states. Namely, the symmetry of a superconducting state is characterized by

$$
\text { Symmetry }_{\mathrm{sc}}=\left[\hat{H}_{\text {hopping }}\right][\hat{\Delta}] \text {, }
$$

where $[\hat{A}]$ indicates the symmetry of $\hat{A}$. This gauge principle does not exist in a conventional BCS-type superconductor in which the information of pairing in real space is irrelevant.

Now we apply the gauge principle and let $\hat{\Delta}_{\mathrm{NN}}$ and $\hat{\Delta}_{\mathrm{NNN}}$ be superconducting order operators associated with $\hat{H}_{d p d, \mathrm{NN}}$ and $\hat{H}_{d p d, \mathrm{NNN}}$, respectively. In a superconducting state that belongs to a pure IR of the original lattice symmetry, we must have

$$
\left[\hat{\Delta}_{\mathrm{NN}}\right]\left[\hat{H}_{d p d, \mathrm{NN}}\right]=\left[\hat{\Delta}_{\mathrm{NNN}}\right]\left[\hat{H}_{d p d, \mathrm{NNN}}\right] .
$$

If we consider a superconducting state that conserves parity, following Eq. (8), we have

$$
\left[\hat{\Delta}_{\mathrm{NN}}\right]=-\left[\hat{\Delta}_{\mathrm{NNN}}\right]
$$

under $\hat{\sigma}_{h}$. Therefore, based on the classification of pairing symmetries in Eq. (5), a parity-conserved superconducting state must be a combination of normal pairing and $\eta$ pairing. If $\hat{\Delta}_{\mathrm{NN}}$ is a normal pairing, we immediately conclude that the state is parity odd, and the superconducting order $\left\langle\hat{\Delta}_{\mathrm{NNN}}\right\rangle$ must be an $\eta$ pairing.

The above analysis can be easily illustrated in real space. As shown in Fig. 4, if superconducting pairing is driven by local $d$ - $p$ hybridization, the superconducting order is a pairing between $d$ and $p$ orbitals, $\Delta_{d p}=\left\langle\hat{d}^{+} \hat{p}^{+}\right\rangle$.

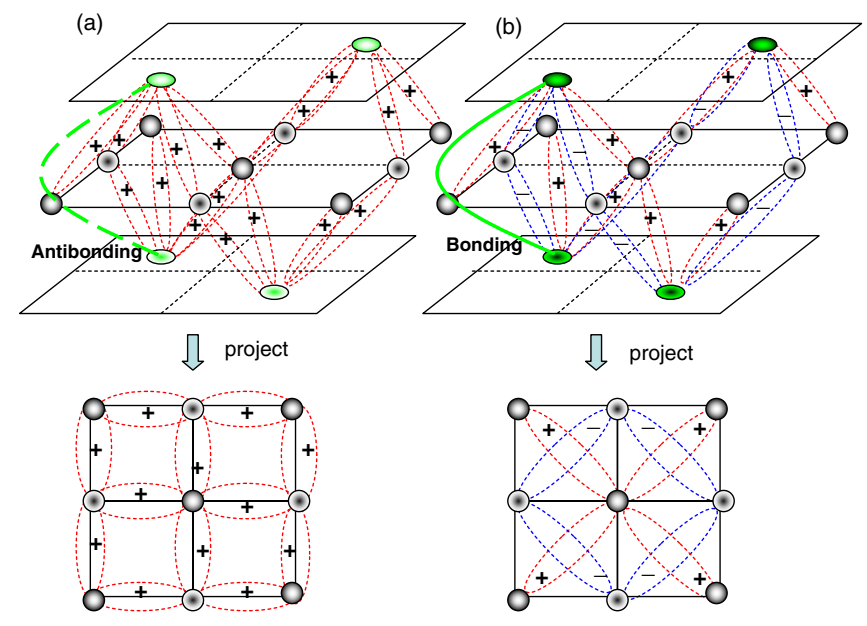

FIG. 4. The NN and NNN $p-d$ local pairing patterns with odd parity are shown in (a) and (b) in the natural gauge. Note that $p$ orbitals of As/Se in (a) form the antibonding states, while those in (b) form the bonding states. We distinguish the two states with different filled green ovals in (a) and (b). The $p-d$ pairings can be projected into effective $d-d$ pairings, as shown in the bottom figures.
A uniform $\left\langle\hat{d}^{+} \hat{p}_{a}^{+}\right\rangle$is parity odd. The NN pairing $\left\langle\hat{\Delta}_{\mathrm{NN}}\right\rangle$ in the effective model must originate from $\left\langle\hat{d}^{+} \hat{p}_{a}^{+}\right\rangle$and thus is also parity odd. The gauge principle can be understood as shown in Figs. 2(c) and 2(d). If we take a new gauge for fermion operators of $p$ orbitals, $\hat{p} \rightarrow-\hat{p}$, in one of the two $\mathrm{As}(\mathrm{Se})$ layers, the antibonding operator $\hat{p}_{a}$ maps to the bonding operator $\hat{p}_{b}$. This gauge mapping exactly transfers the parity between hopping terms and superconducting order parameters.

\section{MEAN-FIELD HAMILTONIAN FOR A PARITY-CONSERVED $S$-WAVE STATE}

The above analysis can be generalized to all effective hoppings. The basic idea is to divide the iron-square lattice into two sublattices. In an odd-parity state, the pairing between two sublattices must be normal pairing, while the pairing within sublattices must be $\eta$ pairing. In an even-parity state, the pairing between two sublattices must be $\eta$ pairing, while the pairing within sublattices must be normal pairing. This means that the pairing between two sublattices must vanish in an even-parity state.

In all of the measured samples of iron-based superconductors, no universal node along the $\Gamma-M$ and $\Gamma-X$ directions on any of the Fermi surfaces was observed [30-39]. These experimental facts require that the superconducting state be in the $A_{1}$ IR of $D_{2 d}$, namely, an $s$ wave viewed at iron sites. Then, the remaining question is about the parity of the state.

In an even-parity $s$-wave state, the normal pairing between two sublattices must vanish. Therefore, the mean-field Hamiltonian for an even-parity $s$-wave state is

$$
H_{m f}^{e}=H_{d d, \mathrm{eff}}+\sum_{\alpha, \beta, k}\left(\delta_{\alpha \beta, n}^{e} \hat{\Delta}_{\alpha \beta, n}(\vec{k})+\text { H.c. }\right),
$$

where $\hat{\Delta}_{\alpha \beta, n}=\hat{d}_{\alpha \uparrow}(\vec{k}) \hat{d}_{\beta \downarrow}(-\vec{k})-d_{\alpha \downarrow}(\vec{k}) \hat{d}_{\beta \uparrow}(-\vec{k})$ and $\alpha, \beta$ label the orbital. In general, the normal pairing order parameters must satisfy

$$
\delta_{\alpha \beta, n}^{e}(\vec{k})=\delta_{\alpha \beta, n}^{e}(\vec{k}+Q) .
$$

All superconducting states derived previously from weakcoupling approaches were considered as even-parity states $[5,8,9,40-43]$. However, as a normal pairing between two sublattices is included, the parity is not conserved. In strong-coupling models [44-47], the superconducting order derived from an NNN antiferromagnetic exchange coupling $J_{2}$, which is a normal pairing within sublattices, satisfies Eq. (13). Therefore, if superconductivity only originates from $J_{2}$, the proposed state is an even-parity $s$-wave state, namely, the $A_{1 g} s$ wave.

This state provides a good understanding of superconducting gaps in iron-based superconductors. However, it cannot explain dual-symmetry characters with both the $s$-wave and $d$-wave types observed in the superconducting state, for example, the spin relaxation $\frac{1}{T_{1} T}$ measured by nuclear magnetic resonance (NMR). A coherent peak 
around $T_{c}$ is expected in a full-gap $s$-wave state even if it is $s^{ \pm}[5,48]$. Experimentally, in an extremely clean sample where the exponential temperature dependence was measured, no coherent peak was observed at $T_{c}$ [49]. For ironchalcogenides [19-22], this state is highly questionable because of the absence of a sign change on Fermi surfaces, so it is hard to explain the possible sign-change evidence from neutron scattering [50]. It is also worth mentioning that many weak-coupling methods suggest that the superconducting state in iron-chalcogenides is a $d$ wave with normal pairing $[18,51,52]$. This state is not consistent with experimental results showing the absence of nodes on high-symmetry lines [37-39] and the presence of strong ferromagnetic NN exchange coupling [53,54]. Nevertheless, this $d$-wave state should be considered as an odd-parity state in the original lattice symmetry because it only includes normal pairing between two sublattices.

A mean-field Hamiltonian to describe the odd-parity $s$-wave state in a 1 -Fe unit cell $\left(A_{1 u} s\right.$ wave) can be generally written as

$H_{m f}^{o}=H_{d d, \text { eff }}+\sum_{\alpha, \beta, k}\left(\delta_{\alpha \beta, n}^{o} \hat{\Delta}_{\alpha \beta, n}(\vec{k})+\delta_{\alpha \beta, \eta}^{o} \hat{\Delta}_{\alpha \beta, \eta}(\vec{k})+\right.$ H.c. $)$,

where $\hat{\Delta}_{\alpha \beta, \eta}=\hat{d}_{\alpha \uparrow}(\vec{k}) \hat{d}_{\beta \downarrow}(-\vec{k}+Q)-\hat{d}_{\alpha \downarrow}(\vec{k}) \hat{d}_{\beta \uparrow}(-\vec{k}+Q)$. In general, the normal- and $\eta$-pairing order parameters satisfy

$$
\begin{gathered}
\delta_{\alpha \beta, n}^{o}(\vec{k})=-\delta_{\alpha \beta, n}^{o}(\vec{k}+Q), \\
\delta_{\alpha \beta, \eta}^{o}(\vec{k})=\delta_{\alpha \beta, \eta}^{o}(\vec{k}+Q) .
\end{gathered}
$$

These equations capture the sign change of superconducting order parameters in momentum space. The sign change here is required by odd-parity symmetry.

While detailed studies will be carried out in the future, the mean-field Hamiltonian captures superconducting gaps in both iron-pnictides and iron-chalcogenides. As the interorbital pairing can be ignored for $s$-wave pairing and the pairing is dominated by $\mathrm{NN}$ and $\mathrm{NNN}$ pairings, the important parameters are

$\delta_{\alpha \alpha, n}^{o} \propto \cos k_{x}+\cos k_{y}, \quad \delta_{\alpha \alpha, \eta}^{o} \propto \cos k_{x} \cos k_{y}$.

Thus, the superconducting gaps on hole pockets are mainly determined by $\delta_{\alpha \alpha, n}^{o}$, and those on electron pockets are mainly determined by $\delta_{\alpha \alpha, \eta}^{o}$.

In the odd-parity $s$-wave state, there is no symmetryprotected node. However, accidental nodes can easily appear. In Fig. 5, we plot numerical results for two cases. Parameters are specified in the caption of the figure. The superconducting gap in the first case is a full gap, while it has gapless nodes on electron pockets in the second case. This may provide an explanation as to why gapless excitations were observed in some materials [5]. The detailed studies will be reported in the future.

The odd-parity $s$-wave state also explains the dualsymmetry character of both the $s$-wave and $d$-wave types (a)

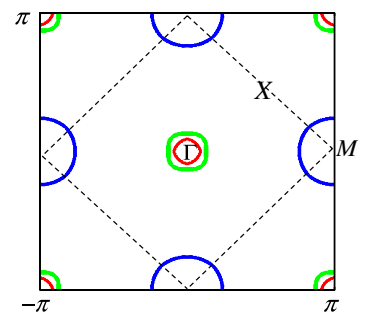

(c)

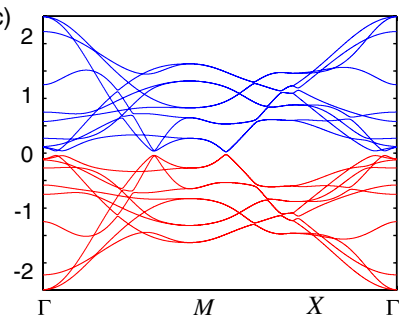

(b)

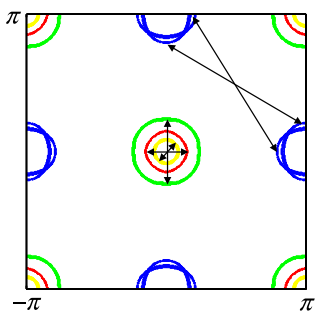

(d)

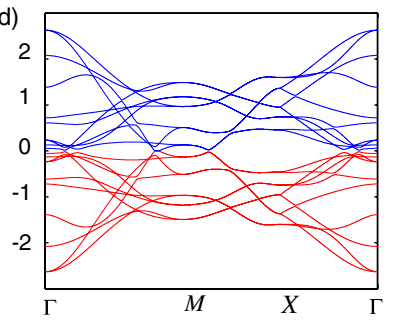

FIG. 5. The Fermi surfaces of a five-orbital model from [25] are shown in (a) and (b). The forms of the hopping terms and hopping parameters can be found in [25]. Here, we only add a chemical potential to tune the Fermi level. We set $\mu=0.1$ and -0.04 in (a) and (b). We define the order parameters in Eq. (16) as $\delta_{11, n}^{o}=\frac{1}{2}\left(\Delta_{11, x}^{N} \cos k_{x}+\Delta_{11, y}^{N} \cos k_{y}\right), \delta_{22, n}^{o}=\frac{1}{2}\left(\Delta_{11, x}^{N} \cos k_{y}+\right.$ $\left.\Delta_{11, y}^{N} \cos k_{x}\right), \delta_{44, n}^{o}=\frac{1}{2} \Delta_{44}^{N}\left(\cos k_{x}+\cos k_{y}\right), \delta_{12, \eta}^{o}=\Delta_{12}^{N N} \sin k_{x} \sin k_{y}$, and $\delta_{\alpha \alpha, \eta}^{o}=\Delta_{\alpha \alpha}^{\mathrm{NN}} \cos k_{x} \cos k_{y}$. The high-symmetry points are shown in (a), and the pairing channels connecting the points on the Fermi surface are denoted by the black lines with arrows. The quasiparticle spectra of the superconductive states for (a) and (b) are shown in (c) and (d). We find that in (c) the spectrum is fully gaped, and (d) has nodes at the electron pockets. The superconducting order parameters are chosen as follows: $\Delta_{11, x}^{N}=\Delta_{11, y}^{N}=0.05, \Delta_{44}^{N}=0.05, \Delta_{11}^{\mathrm{NN}}=0.05, \Delta_{12}^{\mathrm{NN}}=0.05$, and $\Delta_{44}^{N}=-0.1$.

in iron-based superconductors. The $\eta$-pairing $s$-wave order in $D_{2 d}$ essentially is a $d$-wave type order according to $C_{4 v}$, as shown in Fig. 4. For a $d$-wave pairing symmetry, the vanishing of the coherence factor is required by symmetry. Thus, current NMR results really support the odd-parity state.

The Hamiltonian in Eq. (14) cannot be reduced to a translationally invariant Hamiltonian in a $1-\mathrm{Fe}$ unit cell. The 2-Fe unit cell becomes intrinsic. Unique features related to the 2-Fe unit cell should be observed and studied [55]. A detailed study will be carried out in the future.

In summary, we provide the mean-field Hamiltonian for parity-conserved superconducting states. Parity conservation was seriously violated in the past studies. Comparing odd- and even-parity $s$-wave states, we show that the oddparity $s$-wave state can naturally explain many intriguing properties of iron-based superconductors.

\section{SIGNATURE OF THE ODD-PARITY SUPERCONDUCTING STATE}

The fingerprint of the odd-parity state is the negative isospin of $\hat{\sigma}_{h}$, which indicates the sign change of order 


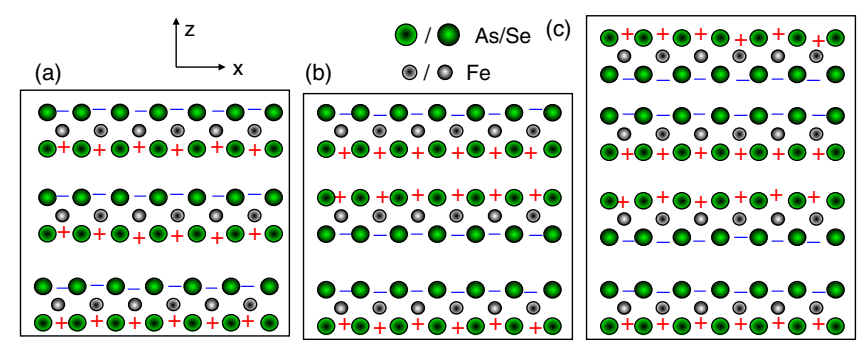

FIG. 6. Sketch of a real-space sign distribution along the $c$ axis for an $\eta$-pairing state in (a) an 11-type structure, (b) a 122-type structure with an odd number of layers, and (c) a 122-type structure with an even number of layers.

parameters between the top and bottom $\mathrm{As}(\mathrm{Se})$ layers. This property was first revealed in the recent constructed effective $S_{4}$ symmetry model based on the $2-\mathrm{Fe}$ unit cell $[16,29,56]$. However, as the $S_{4}$ model is a simplified effective model based on two effective orbitals, the parity characters were not revealed.

The odd parity indicates that a superconducting single Fe-As(As) trilayer is a $\pi$ junction along the $c$ axis, which can be measured in a single-crystal material, as shown in Fig. 6: A uniform superconducting state is characterized by a sign change between the top and bottom surfaces along the $c$ axis in the 11-type structure, while in the 122-type structure, the sign change is only present when the number of layers is odd.

\section{DISCUSSION}

In the history of condensed-matter physics, a new quantum state of condensed matter is not easily obtained by solving a model. Here, we use fundamental principles to show that an odd-parity state can naturally occur in ironbased superconductors, and we suggest smoking-gun experiments to detect or falsify this occurrence. With the microscopic understanding proposed here, detection of the odd-parity state can have a tremendous impact on high- $T_{c}$ mechanisms for iron-based superconductors and other high- $T_{c}$ superconductors.

In the past five years, much research based on effective models suggests that pairing symmetries in iron-based superconductors are very fragile. Those studies essentially suggest that principles to understand the robustness of superconductivity and pairing symmetry are still missing in our standard approach. The results in this paper demonstrate that previous studies did not correctly take parity conservation and hidden symmetry in effective models into account and mishandled symmetry and gauge principles. In the past, the effective Hamiltonian was viewed in the symmetry group $D_{4 h}$ at iron sites rather than the original lattice symmetry $G$. We note that if $\hat{\sigma}_{h}$ is set to $1, G$ is equivalent to $D_{4 h}$. However, because of the antibonding $p$-orbital states, the effective Hamiltonian does not represent the correct symmetry of the original lattice in a natural gauge setting. It will be interesting to see how the missing pieces can be properly implemented in our standard methods.

Our results suggest that the gauge principle needs to be properly implemented when we derive the effective Hamiltonian in order to simplify a complex system. The correct physics can only be understood after the hidden gauge is revealed. For an order parameter that is momentum dependent, this gauge information is critical. The gauge principle becomes very important for us to search for new physics in other complex electron systems.

The odd-parity state also suggests the importance of correlated electron physics. It is believed that sign-change superconducting order is inevitable in a superconducting state of strongly correlated electron systems because of the existence of strong repulsive interactions. This principle is violated in a parity-even $s$-wave state. A measurement of the parity-odd $s$-wave state can provide strong support for this principle.

The odd-parity $s$-wave state closely resembles the $d$-wave state in a $\mathrm{Cu}-\mathrm{O}$ plane of cuprates. We expect that there must be an identical mechanism to select signchanged superconducting order in both materials. From Fig. 4(b), one can see that the $\eta$-pairing part in the oddparity state can be viewed as two $d$-wave states formed in two sublattices, a direct analogy to the $d$ wave in cuprates.

This study opens a promising new direction for the research in iron-based superconductors and suggests that the physics in these materials is deeper and much more inspiring than what we realized before. This research leaves us with many unanswered questions. Some clarifications are in order. First, what is the relationship between magnetism and superconductivity? One can see that the collinear antiferromagnetic (C-AFM) state [57] observed in iron-pnictides has odd parity. This characteristic may partially answer why superconductivity and C-AFM order can coexist in the phase diagram. Second, what are the other unique properties in an odd-parity $s$-wave state? It is known that an odd-parity $p$-wave state displays many unique properties. Third, what is the relationship between nematism and superconductivity? Nematism breaks rotational symmetry and is observed at high temperatures [58]. Fourth, how robust is an odd-parity state in response to impurity? This study points out that there are three possible scenarios related to parity in superconducting states: even, odd, or broken. Experiments proposed here will finally nail down the truth. All of the previous studies took even parity for granted without knowing that the parity was actually broken in the proposed states. However, a parity-breaking superconducting state is also an interesting state to explore.

In summary, using symmetry and gauge principles, we showed that iron-based superconductors are unified into an odd-parity $s$-wave superconducting state. Provided that the essential physics is through the $d$ - $p$ hybridization, we show that in an effective model based on $d$ orbitals, superconducting states studied in the past violate parity conservation. The 
existence of the odd-parity state can have a tremendous impact on high- $T_{c}$ superconducting mechanisms.

\section{ACKNOWLEDGMENTS}

The author acknowledges N. N. Hao for useful discussions and for providing the numerical results for superconducting gaps, and $\mathrm{X}$. X. Wu for providing the figure for the distribution of the $p$ orbital in LDA calculations. The author wants to thank H. Ding, P. Coleman, D. Scalapino, T. Xiang, X. Dai, and D.L. Feng for useful discussions. This work is supported by the Ministry of Science and Technology of China 973 program (Grant No. 2012CB821400) and Grant No. NSFC-1190024.

[1] Y. Kamihara, T. Watanabe, M. Hirano, and H. Hosono, Iron-Based Layered Superconductor $\mathrm{La}\left[\mathrm{O}_{1-x} \mathrm{~F}_{x}\right] \mathrm{FeAs}(x=0.05-0.12) \quad$ with $\quad T_{c}=26 \mathrm{~K}$, J. Am. Chem. Soc. 130, 3296 (2008).

[2] X.H. Chen, T. Wu, G. Wu, R. H. Liu, H. Chen, and D. F. Fang, Superconductivity at $43 \quad K$ in $\mathrm{SmFeAsO}_{1-x} \mathrm{~F}_{x}$, Nature (London) 453, 761 (2008).

[3] G. F. Chen, Z. Li, D. Wu, G. Li, W.Z. Hu, J. Dong, P. Zheng, J.L. Luo, and N. L. Wang, Superconductivity at $41 \mathrm{~K}$ and Its Competition with Spin-Density-Wave Instability in Layered $\mathrm{CeO}_{1-x} \mathrm{~F}_{x} \mathrm{FeAs}$ Phys. Rev. Lett. 100, 247002 (2008).

[4] D.C. Johnston, The Puzzle of High Temperature Superconductivity in Layered Iron Pnictides and Chalcogenides, Adv. Phys. 59, 803 (2010).

[5] P. J. Hirschfeld, M. M. Korshunov, and I. I. Mazin, Gap Symmetry and Structure of Fe-Based Superconductors, Rep. Prog. Phys. 74, 124508 (2011).

[6] C. C. Tsuei and J. R. Kirtley, Pairing Symmetry in Cuprate Superconductors, Rev. Mod. Phys. 72, 969 (2000).

[7] P. W. Anderson, Structure of "Triplet" Superconducting Energy Gaps, Phys. Rev. B 30, 4000 (1984).

[8] K. Kuroki, Seiichiro Onari, Ryotaro Arita, Hidetomo Usui, Yukio Tanaka, Hiroshi Kontani, and Hideo Aoki, Unconventional Pairing Originating from the Disconnected Fermi Surfaces of Superconducting $\mathrm{LaFeO}_{1-x} \mathrm{~F}_{x}$, Phys. Rev. Lett. 101, 087004 (2008).

[9] S. Graser, A. F. Kemper, T. A. Maier, H.-P. Cheng, P. J. Hirschfeld, and D. J. Scalapino, Spin Fluctuations and Superconductivity in a Three-Dimensional Tight-Binding Model for $\mathrm{BaFe}_{2} \mathrm{As}_{2}$, Phys. Rev. B 81, 214503 (2010).

[10] H. Eschrig and K. Koepernik, Tight-Binding Models for the Iron-Based Superconductors, Phys. Rev. B 80, 104503 (2009).

[11] P. A. Lee and X. -G. Wen, Spin-Triplet p-Wave Pairing in a Three-Orbital Model for Iron Pnictide Superconductors, Phys. Rev. B 78, 144517 (2008).

[12] M. Daghofer, A. Nicholson, A. Moreo, and E. Dagotto, Three Orbital Model for the Iron-Based Superconductors, Phys. Rev. B 81, 14511 (2010).

[13] S. Raghu, X. L. Qi, C. X. Liu, D. J. Scalapino, and S.C. Zhang, Minimal Two-Band Model of the Superconducting Iron Oxypnictides, Phys. Rev. B 77, 220503 (2008).
[14] C. Yang, $\eta$ Pairing and Off-Diagonal Long-Range Order in a Hubbard Model, Phys. Rev. Lett. 63, 2144 (1989).

[15] C. Yang and S. Zhang, $\mathrm{SO}_{4}$ Symmetry in a Hubbard Model, Mod. Phys. Lett. B 04, 759 (1990).

[16] J. Hu and N. Hao, $S_{4}$ Symmetric Microscopic Model for Iron-Based Superconductors, Phys. Rev. X 2, 021009 (2012).

[17] Y. Gao, W.P. Su, and J.X. Zhu, Interorbital Pairing and Its Physical Consequences for Iron Pnictide Superconductors, Phys. Rev. B 81, 104504 (2010).

[18] M. Khodas and A. V. Chubukov, Interpocket Pairing and Gap Symmetry in Fe-Based Superconductors with Only Electron Pockets, Phys. Rev. Lett. 108, 247003 (2012).

[19] J. Guo, S. Jin, G. Wang, S. Wang, K. Zhu, T. Zhou, M. He, and $\mathrm{X}$. Chen, Superconductivity in the Iron Selenide $\mathrm{K}_{x} \mathrm{Fe}_{2} \mathrm{Se}_{2}(0 \leq x \leq 1.0)$, Phys. Rev. B 82, 180520(R) (2010).

[20] S. He et al., Phase Diagram and High Temperature Superconductivity at $65 K$ in Tuning Carrier Concentration of Single-Layer FeSe Films, arXiv:1207.6823.

[21] D. Liu et al., Electronic Origin of High-Temperature Superconductivity in Single-Layer FeSe Superconductor, Nat. Commun. 3, 931 (2012).

[22] S. Y. Tan et al., Interface-Induced Superconductivity and Strain-Dependent Spin Density Wave in FeSe/SrTiO3 Thin Films, arXiv:1301.2748.

[23] M. H. Fischer, F. Loder, and M. Sigrist, Superconductivity and Local Noncentrosymmetricity in Crystal Lattices, Phys. Rev. B 84, 184533 (2011).

[24] T. Miyake, K. Nakamura, R. Arita, and M. Imada, Comparison of Ab Initio Low-Energy Models for LaFePo, LaFeAsO, $\mathrm{BaFe}_{2} \mathrm{As}_{2}$, LiFeAs, FeSe and FeTe: Electron Correlation and Covalency, J. Phys. Soc. Jpn. 79, 044705 (2010).

[25] S. Graser, T. A. Maier, P. J. Hirschfeld, and D. J. Scalapino, Near-Degeneracy of Several Pairing Channels in Multiorbital Models for the Fe Pnictides, New J. Phys. 11, 025016 (2009).

[26] F. Ma and Z.-Y. LU, Iron-Based Layered Compound LaFeAsO Is an Antiferromagnetic Semimetal, Phys. Rev. B 78, 033111 (2008).

[27] D. J. Singh, Electronic Structure and Doping in $\mathrm{BaFe}_{2} \mathrm{As}_{2}$ and LiFeAs: Density Functional Calculations, Phys. Rev. B 78, 094511 (2008).

[28] E. Berg, S. A. Kivelson, and D. J. Scalapino, A Twisted Ladder: Relating the Fe Superconductors to the High- $T_{c}$ Cuprates, New J. Phys. 11, 085007 (2009).

[29] J. A Hu, Short Review of the $S_{4}$ Symmetric Microscopic Model for Iron-Based High Temperature Superconductors, arXiv:1208.6201.

[30] L. Zhao et al., Multiple Nodeless Superconducting Gaps in $\left(\mathrm{Ba}_{0.6} \mathrm{~K}_{0.4}\right) \mathrm{Fe}_{2} \mathrm{As}_{2}$ Superconductor from Angle-Resolved Photoemission Spectroscopy, Chin. Phys. Lett. 25, 4402 (2008).

[31] H. Ding and et al., Observation of Fermi-Surfacedependent Nodeless Superconducting Gaps in $\mathrm{Ba}_{0.6} \mathrm{~K}_{0.4} \mathrm{Fe}_{2} \mathrm{As}_{2}$, Europhys. Lett. 83, 47001 (2008).

[32] L. Wray et al., Momentum Dependence of Superconducting Gap, Strong-Coupling Dispersion Kink, and Tightly Bound Cooper Pairs in the High-T 
$(\mathrm{Sr}, \mathrm{Ba})_{1-x}(\mathrm{~K}, \mathrm{Na})_{x} \mathrm{Fe}_{2} \mathrm{As}_{2}$ Superconductors, Phys. Rev. B 78, 184508 (2008).

[33] H. Ding et al., Electronic Structure of Optimally Doped Pnictide $\mathrm{Ba}_{0.6} \mathrm{~K}_{0.4} \mathrm{Fe}_{2} \mathrm{As}_{2}$ : A Comprehensive AngleResolved Photoemission Spectroscopy Investigation, J. Phys. Condens. Matter 23, 135701 (2011).

[34] L. Ding et al., Nodeless Superconducting Gap in ElectronDoped $\mathrm{BaFe}_{1.9} \mathrm{Ni}_{0.1} \mathrm{As}_{2}$ Probed by Quasiparticle Heat Transport, New J. Phys. 11, 093018 (2009).

[35] K. Nakayama et al., Universality of Superconducting Gaps in Overdoped $\mathrm{BaFe}_{1.9} \mathrm{Ni}_{0.1} \mathrm{As}_{2}$ Observed by AngleResolved Photoemission Spectroscopy, Phys. Rev. B 83, 020501(R) (2011).

[36] Y. Zhang et al., Nodeless Superconducting Gap in $\mathrm{A}_{x} \mathrm{Fe}_{2} \mathrm{Se}_{2} \quad(A=K, C s)$ Revealed by Angle-Resolved Photoemission Spectroscopy, Nat. Mater. 10, 273 (2011).

[37] Y. Zhang et al., Out-of-Plane Momentum and Symmetry-Dependent Energy Gap of the Pnictide $\mathrm{Ba}_{0.6} \mathrm{~K}_{0.4} \mathrm{Fe}_{2} \mathrm{As}_{2}$ Superconductor Revealed by AngleResolved Photoemission Spectroscopy, Phys. Rev. Lett. 105, - (2010).

[38] X.-P. Wang et al., Observation of an Isotropic Superconducting Gap at the Brillouin Zone Center of $\mathrm{Tl}_{0.63} \mathrm{~K}_{0.37} \mathrm{Fe}_{1.78} \mathrm{Se}_{2}$, Europhys. Lett. 99, 67001 (2012).

[39] M. Xu et al., Angle-Resolved Photoemission Evidence of s-Wave Superconducting Gap in $\mathrm{K}_{x} \mathrm{Fe}_{2-y} \mathrm{Se}_{2}$ Superconductor, Phys. Rev. B 85, 220504(R) (2012).

[40] I. I. Mazin, D. J. Singh, M. D. Johannes, and M. H. Du, Unconventional Superconductivity with a Sign Reversal in the Order Parameter of $\mathrm{LaFeAsO}_{1-x} \mathrm{~F}_{x}$, Phys. Rev. Lett. 101, 57003 (2008).

[41] F. Wang, H. Zhai, Y. Ran, A. Vishwanath, and D.-H. Lee, Functional Renormalization-Group Study of the Pairing Symmetry and Pairing Mechanism of the FeAs-Based High-Temperature Superconductor, Phys. Rev. Lett. 102, 47005 (2009).

[42] A. V. Chubukov, Renormalization Group Analysis of Competing Orders and the Pairing Symmetry in $\mathrm{Fe}$ Based Superconductors, Physica (Amsterdam) 469, 640 (2009).

[43] R. Thomale, C. Platt, J. P. Hu, C. Honerkamp, and B. A. Bernevig, Functional Renormalization-Group Study of the Doping Dependence of Pairing Symmetry in the Iron Pnictide Superconductors, Phys. Rev. B 80, 180505(R) (2009).

[44] K. J. Seo, B. A. Bernevig, and J. P. Hu, Pairing Symmetry in a Two-Orbital Exchange Coupling Model of Oxypnictides, Phys. Rev. Lett. 101, 206404 (2008).

[45] X. Lu, C. Fang, W.-F. Tsai, Y. Jiang, and J. Hu, s-Wave Superconductivity with Orbital-Dependent Sign Change in
Checkerboard Models of Iron-Based Superconductors, Phys. Rev. B 85, 54505 (2012).

[46] C. Fang, Y.-L. Wu, R. Thomale, B. A. Bernevig, and J. $\mathrm{Hu}$, Robustness of $s$-Wave Pairing in ElectronOverdoped $\mathrm{A}_{1-y} \mathrm{Fe}_{2-x} \mathrm{Se}_{2}$, Phys. Rev. X 1, 011009 (2011).

[47] R. Yu, P. Goswami, Q. Si, P. Nikolic, and J.-X. Zhu, Pairing Strength and Symmetries of $(K, T l)$ FexSe2 in Comparison with Iron Pnictides., arXiv:1103.3259.

[48] M. M. Parish, J. P. Hu, and B. A. Bernevig, Experimental Consequences of the $s$-Wave $\cos \left(k_{x}\right) \cos \left(k_{y}\right)$ Superconductivity in the Iron Pnictides, Phys. Rev. B 78, 144514 (2008).

[49] Z. Li, D. L. Sun, C. T. Lin, Y. H. Su, J. P. Hu, and Guo-qing Zheng, Nodeless Energy Gaps of Single-Crystalline $\mathrm{Ba}_{0.68} \mathrm{~K}_{0.32} \mathrm{Fe}_{2} \mathrm{As}_{2}$ as Seen via ${ }^{75}$ As NMR, Phys. Rev. B 83, 140506 (2011).

[50] J. T. Park et al., Magnetic Resonant Mode in Superconducting $\mathrm{Rb}_{2} \mathrm{Fe}_{4} \mathrm{Se}_{5}$, Phys. Rev. Lett. 107, 177005 (2011).

[51] T. Maier, S. Graser, P. Hirschfeld, and D. Scalapino, $d$-Wave Pairing from Spin Fluctuations in the $\mathrm{K}_{x} \mathrm{Fe}_{2-y} \mathrm{Se}_{2}$ Superconductors, Phys. Rev. B 83, 100515 (R) (2011).

[52] F. Wang, Fan Yang, Miao Gao, Zhong-Yi Lu, Tao Xiang, and Dung-Hai Lee, The Electron Pairing of $\mathrm{K}_{x} \mathrm{Fe}_{2-y} \mathrm{Se}_{2}$, Europhys. Lett. 93, 57003 (2011).

[53] M. Wang et al., Spin Waves and Magnetic Exchange Interactions in Insulating $\mathrm{Rb}_{0.89} \mathrm{Fe}_{1.58} \mathrm{Se}_{2}$, Nat. Commun. 2, 580 (2011).

[54] J. Hu, B. Xu, W. Liu, N.-N. Hao, and Y. Wang, Unified Minimum Effective Model of Magnetic Properties of Iron-Based Superconductors, Phys. Rev. B 85, 144403 (2012).

[55] V. Brouet, M. Fuglsang Jensen, Ping-Hui Lin, A. TalebIbrahimi, P. Le Fèvre, F. Bertran, Chia-Hui Lin, Wei Ku, A. Forget, and D. Colson, Impact of the Two Fe Unit Cell on the Electronic Structure Measured by ARPES in Iron Pnictides, Phys. Rev. B 86, 075123 (2012).

[56] T. Ma, H.-Q. Lin, and J. Hu, Quantum Monte Carlo Study of Pairing Symmetry and Correlation in Iron-Based Superconductors, Phys. Rev. Lett. 110, 107002 (2013).

[57] P. Dai, J. Hu, and E. Dagotto, Magnetism and Its Microscopic Origin in Iron-Based High-Temperature Superconductors, Nat. Phys. 8, 709 (2012).

[58] I. R. Fisher, L. Degiorgi, and Z.X. Shen, In-Plane Electronic Anisotropy of Underdoped "122" Fe-Arsenide Superconductors Revealed by Measurements of Detwinned Single Crystals, Rep. Prog. Phys. 74, 124506 (2011). 